\title{
Simvastatin protects Sertoli cells against cisplatin cytotoxicity through enhanced gap junction intercellular communication
}

\author{
LINGZHI WANG ${ }^{1,2^{*}}$, JIANXIN PENG $^{3 *}$, HUANSEN HUANG $^{1}$, QIN WANG $^{2}$, MEILING YU $^{4}$ and LIANG TAO ${ }^{2}$ \\ ${ }^{1}$ Department of Anaesthesia, The Second Affiliated Hospital, Guangzhou Medical University, Guangzhou 510260; \\ ${ }^{2}$ Department of Pharmacology, Zhongshan School of Medicine, Sun Yat-Sen University, Guangzhou 510080; \\ ${ }^{3}$ Department of Hepatobiliary Surgery, Guangdong Province Traditional Chinese Medical Hospital, Guangzhou 510120; \\ ${ }^{4}$ Department of Pharmacy, The First Affiliated Hospital, Bengbu Medical College, Bengbu, Anhui 233004, P.R. China
}

Received June 16, 2015; Accepted July 9, 2015

DOI: $10.3892 /$ or.2015.4192

\begin{abstract}
Cisplatin, an important chemotherapeutic agent against testicular germ cell cancer, induces testicular toxicity on Leydig and Sertoli cells, leading to serious side-effects such as azoospermia and infertility. In a previous study, it was found that simvastatin enhanced the sensitivity of Leydig tumor cells to chemotherapeutic toxicity through the enhancement of gap junction functions. In the present study, the effect of simvastatin on the sensitivity of normal Sertoli cells to cisplatin and the role of gap junctions in such effects was investigated. The results showed that, simvastatin attenuated cisplatin toxicity only when cells exhibited high-density culture where gap junctional formation was possible. When gap junction function was decreased by the gap junction inhibitor or by siRNA targeting connexin 43 , the protective effect of simvastatin to cisplatin toxicity was substantially attenuated. Simvastatin also enhanced gap junction functions between Sertoli cells. This effect was mediated by the reduction of PKC-mediated connexin phosphorylation, thereby increasing connexin 43 membrane localization. Thus, simvastatin-induced enhancement of gap junction-mediated intercellular communication attenuated cisplatin toxicity on Sertoli cells. This result indicated that enhancement of gap
\end{abstract}

Correspondence to: Professor Liang Tao, Department of Pharmacology, Zhongshan School of Medicine, Sun Yat-Sen University, No.74, Zhongshan Road 2, Guangzhou 510080, P.R. China

E-mail: taol@mail.sysu.edu.cn

Professor Huansen Huang, Department of Anaesthesia, The Second Affiliated Hospital, Guangzhou Medical University, 250 Changgang East Road, Guangzhou 510260, P.R. China

E-mail: huanghs5480@163.com

*Contributed equally

Abbreviations: GJIC, gap junctional intercellular communication; TPA, 12-O-tetradecanoylphorbol 13 -acetate

Key words: cisplatin, Sertoli cell, simvastatin, gap junction, connexin junction function by simvastatin may have bilateral beneficial effects on cisplatin-based chemotherapy, enhancing cisplatin killing on cancer while ameliorating the reproduction toxicity.

\section{Introduction}

The incidence of testicular cancer has been on the increase over the last few decades due to increasing environmental toxicants (1). Testicular germ cell cancer is a curable cancer and cisplatin is the potent chemotherapeutic agent used to combat this disease. However, cisplatin also affects germ, Sertoli and Leydig cells during chemotherapy, which results in a $30-50 \%$ infertility rate for testicular cancer survivors $(2,3)$. Identification of a strategy to attenuate cisplatin-induced testicular toxicity without affecting the killing of cancer cells largely improves long-term quality of life for testicular cancer patients.

Gap junction-mediated intercellular communication (GJIC) is reported to have enhancing effects on the toxicity of chemotherapeutic agents such as cisplatin and etoposide $(4,5)$ to cancer cells. Gap junction (GJ) is formed by the docking of two hemichannels from neighbouring cells. Each hemichannel is composed of six homogenous or heterogeneous connexins (Cxs). The connexin family comprises 20 members in mouse and 21 in human, in which connexin 43 (Cx43) is a predominant $\mathrm{Cx}$ member that expressed in human testicular tissues and testicular cancer cells $(6,7)$.

Gap junctions provide a direct pathway for the rapid inter-cytoplasmic diffusion of hydrophilic metabolites and signaling molecules between adjacent cells. Accumulated evidence suggests that various death signals triggered by antitumor agents propagated through gap junctions from target cells to neighbouring cells, thereby promoting antineoplastic efficacy in cancer cells $(4,8)$. This 'bystander' effect is not limited to death signals. Previous findings have demonstrated that the protective signal may spread through gap junctions between normal cells in response to oxidative stress and ischemic injury (9-11). Hong et al reported that gap junctions between testicular cancer cells communicated predominantly toxic effects and increasing cisplatin toxicity, while gap junctions between normal testicular cells communicated predominantly protective effects, decreasing cisplatin toxicity to Sertoli and Leydig cells (12). This finding suggests that an 
increase in GJIC enhances cisplatin-induced toxic effect on cancer cells while protecting normal testicular cells against cisplatin-induced injury.

Statins are competitive inhibitors of 3-hydroxy-3-methylglutaryl-coenzyme A (HMG-CoA) reductase. They reduce plasma cholesterol levels and are widely used for cardiovascular disease resulting from hypercholesterolemia. There is considerable interest concerning the therapeutic utilization of statins in antineoplastic and antioxidation treatment $(13,14)$. Our previous results showed that simvastatin increased GJ function in Leydig tumor cells and increased etoposide cytotoxicity to these cells (15). Nevertheless, to the best of our knowledge, there is no evidence to demonstrate the effect of simvastatin on GJIC between normal testicular cells and whether this interaction is involved in the effect of simvastatin on the response of testicular normal cells to chemotherapeutic compounds.

In the present study, we confirmed that GJ protected TM4 Sertoli cells against cisplatin toxicity. Simvastatin attenuated cisplatin toxicity, which was decreased when GJIC was inhibited by a chemical inhibitor or Cx43-siRNA. Moreover, we revealed that simvastatin induced enhancing effects on the gap junction through the downregulation of phosphorylated Cx43. Our results indicated that the inhibitory effect of simvastatin on cisplatin toxicity in TM4 cells may be attributed to an increase of GJIC by decreasing Cx43 phosphorylation. Simvastatin, a sensitizer of testicular tumor cells to the antineoplastic agent demonstrated in our previous study, protects normal testicular cells from cisplatin toxicity.

\section{Materials and methods}

Materials. Cisplatin was purchased from Sigma-Aldrich, St. Louis, MO, USA, and was dissolved in phosphate-buffered saline (PBS) for a stock concentration. Cell culture reagents were all obtained from Invitrogen Life Technologies (Carlsbad, CA, USA). Simvastatin was purchased from Sigma-Aldrich. Simvastatin $(20 \mathrm{mg})$, which is a lactone prodrug, was diluted in $0.5 \mathrm{ml} 100 \%$ ethanol, followed by the addition of $0.75 \mathrm{ml}$ $0.1 \mathrm{M} \mathrm{NaOH}$ and heating at $50^{\circ} \mathrm{C}$ for $2 \mathrm{~h}$. The solution was neutralized with $0.1 \mathrm{M} \mathrm{HCl}$ to $\mathrm{pH} 7.2$ and adjusted with PBS to a final concentration of $5 \mathrm{mM}$, sterilized by filtration and aliquots were stored at $-20^{\circ} \mathrm{C}$.

Cell lines and cell culture. The mouse Sertoli cell line (TM4) was obtained from the American Type Culture Collection (ATCC; Manassas, VA, USA). TM4 cells were cultured in Dulbecco's modified Eagle's medium (DMEM)/F-12 supplemented with $5 \%$ horse serum and $2.5 \%$ fetal bovine serum, $100 \mathrm{U} / \mathrm{ml}$ penicillin and $100 \mathrm{~g} / \mathrm{ml}$ streptomycin. The cells were grown at $37^{\circ} \mathrm{C}$ in an atmosphere of $5 \% \mathrm{CO}_{2}$ in air.

RNA interference. RNA interference of Cx43 expression was performed by stable cell lines endogenously expressing shRNA. pSilencer 2.1-U6 neo plasmids expressing shRNA targeting Cx43 (target sequence, 5'-GCTCACGTGTTCTATGTGA-3') or pSilencer 2.1-U6 negative control plasmids (both from Ambion Europe, UK) were transfected into cells, and stable cell lines were selected by $0.1 \mathrm{mg} / \mathrm{ml} \mathrm{G} 418$ and identified by western blotting and by parachute dye transfer assay (12).
Standard colony-forming assay. Cell survival was assayed by a standard colony-forming assay, adapted for use at high and low cell density, corresponding to conditions in which junctional channel formation was permitted or not, respectively (8). In the high-density condition, the cells were seeded at $3 \times 10^{4}$ cells $/ \mathrm{cm}^{2}$ thus that cultures were $70-100 \%$ confluent at the time of cisplatin exposure. The cells were treated with cisplatin for $1 \mathrm{~h}$ in the dark, then washed with PBS, harvested by trypsinization, counted, diluted and seeded in 6-well dishes (100 cells/well). Colony formation was assessed 5-7 days later by fixation and staining with crystal violet. Colonies containing $\geq 50$ cells were scored. In the low-density condition, the cells were directly seeded at a density of 500 cells $/ \mathrm{cm}^{2}$ in 6 -well plates and treated with cisplatin for $1 \mathrm{~h}$ after attachment. The plates were rinsed and assessed for colony formation as previously described. Colony formation was normalized to the colony-forming efficiency of non-cisplatin-treated cells. There was no significant difference in the plating efficiency between the low- and high-density cultures in the untreated samples (data not shown). Simvastatin was added to the cultural medium $3 \mathrm{~h}$ prior to cisplatin treatment.

Evaluation of the GJIC capacity. To evaluate GJIC capacity, we performed 'Parachute' dye-coupling assay as described by Goldberg et al and Koreen et al (16). Donor and receiver TM4 cells were grown to $80-90 \%$ confluency. The donor cells were double-labeled with $10 \mu \mathrm{M} \mathrm{CM}$-DiI, a membrane dye that cannot spread to coupled cells, and $10 \mu \mathrm{M}$ calcein-AM (both from Invitrogen Life Technologies), which is converted intercellularly into the gap junction-permeable dye calcein. The donor cells were washed, trypsinized and then resuspended with serum-free medium and seeded onto the receiver cells at a 1:150 donor/receiver ratio. Simvastatin was added in the donor cell suspension at the same time. The donor cells were allowed to attach for $4 \mathrm{~h}$ at $37^{\circ} \mathrm{C}$ to form gap junctions with receiver cells, followed by examination with a fluorescence microscope. For each experimental condition, the average number of receiver cells containing dye calcein around one donor cell was determined and normalized to that of the control.

Western blotting. Protein extractions were carried out by direct dissolution of cells in protein lysis buffer containing protease inhibitors (Sigma-Aldrich). Protein concentrations were determined using Bio-Rad Dc protein assay reagent (Bio-Rad, Hercules, CA, USA). Approximately $20 \mu \mathrm{g}$ of lysate protein was separated on the $10 \%$ SDS-PAGE gel, then electrically transferred to polyvinylidene difluoride (PVDF) membranes (Bio-Rad) and blocked with 5\% skim milk TBST. Individual membranes were probed with monoclonal antiCx43 antibody produced in mouse (1:4,000; Sigma-Aldrich) and monoclonal antibodies against p-Cx43 (ser368) (Cell Signaling Technology, Beverly, MA, USA). The membranes were incubated with horseradish peroxidase (HRP)-conjugated secondary antibodies (GE Healthcare, Pittsburgh, PA, USA). The immunoreactive bands were visualized by ECL plus western blotting detection system (GE Healthcare). Blots were re-probed with an anti $\beta$-tubulin antibody (Sigma-Aldrich) and developed in an identical manner for assessing $\beta$-tubulin protein levels. The intensities were quantified by Quantity One software on GS-800 densitometer (Bio-Rad). 
Immunofluorescent staining. TM4 cells were plated on glass coverslips in 24-well plates and treated with $10 \mu \mathrm{M}$ simvastatin when $80 \%$ confluency was reached. The cells were fixed in $4 \%$ paraformaldehyde at room temperature, washed and blocked with 5\% BSA for $30 \mathrm{~min}$ at room temperature. This process was followed by incubation with monoclonal anti-mouse $\mathrm{Cx} 43$ antibody (1:1,500; Sigma-Aldrich) dissolved in 5\% BSA. The cells were incubated with the goat anti-mouse secondary antibody conjugated with fluorescein isothiocyanate (1/400; Invitrogen Life Technologies), washed and mounted with a solution containing P-phenylenediamine + glycerol + PBS at the proportions 29:9:1. Fluorescence was viewed and images were captured with a fluorescence microscope.

PKC activity assay. The total protein kinase $\mathrm{C}$ activity of TM4 cells was determined using the PepTag non-radioactive PKC assay kit (Promega, Madison, WI, USA), according to the manufacturer's instructions. The PepTag assay uses fluorescent peptide substrate which is highly specific for PKC. Phosphorylation of this substrate by PKC alters the peptides net charge from +1 to -1 . This change in the net charge of the substrate allows the phosphorylated and non-phosphorylated versions of the substrate to be rapidly separated on an agarose gel $(0.8 \%)$. The phosphorylated species migrates towards the positive electrode, while the non-phosphorylated substrate migrates towards the negative electrode. The bands were then visualized under UV light and excised from the gel, and heated at $95^{\circ} \mathrm{C}$ until the gel was sectioned. The bands were melted and mixed with Gel solubilization solution, glacial acetic acid and distilled water. Absorbency was read at $570 \mathrm{~nm}$ using a spectrophotometer in a 96-well plate.

Statistical analysis. Data were statistically analyzed using the unpaired Student's t-test at a significance level of $\mathrm{P}<0.05$. Data are presented as means \pm SEM unless otherwise indicated using SigmaPlot (Jandel Scientific, San Rafael, CA, USA).

\section{Results}

Gap junctions in TM4 cells attenuate cisplatin cytotoxicity. TM4 cells expressing Cx43 were cultured under conditions where gap junction formation was not possible (low-density; 100 cells $/ \mathrm{cm}^{2}$; cells not in direct contact with each other) and where gap junction formation was possible (high-density; $3 \times 10^{4}$ cells $/ \mathrm{cm}^{2}$ ). The cells were treated with cisplatin for $1 \mathrm{~h}$ and then cell survival was assessed as described in Materials and methods. As shown in Fig. 1A, treatment with cisplatin reduced the clonogenic survival of TM4 cell at low and high density in a concentration-dependent manner. The toxic effect of cisplatin was much less at a high cell density than at a low cell density.

To investigate the role of GJIC in the density-dependence of cisplatin response in TM4 cells, the gap junction function was regulated by two methods: pharmacological inhibition on junctional channels by TPA and downregulation of $\mathrm{Cx} 43$ expression by siRNA. TPA is a well-recognized chemical inhibitor for $\mathrm{Cx} 43$ channels. Fig. 2 shows that, TPA significantly suppressed the dye spread through junctional channels in TM4 cells. At high cell density, pretreatment of TM4 cells with TPA increased cisplatin toxicity, and manifested a
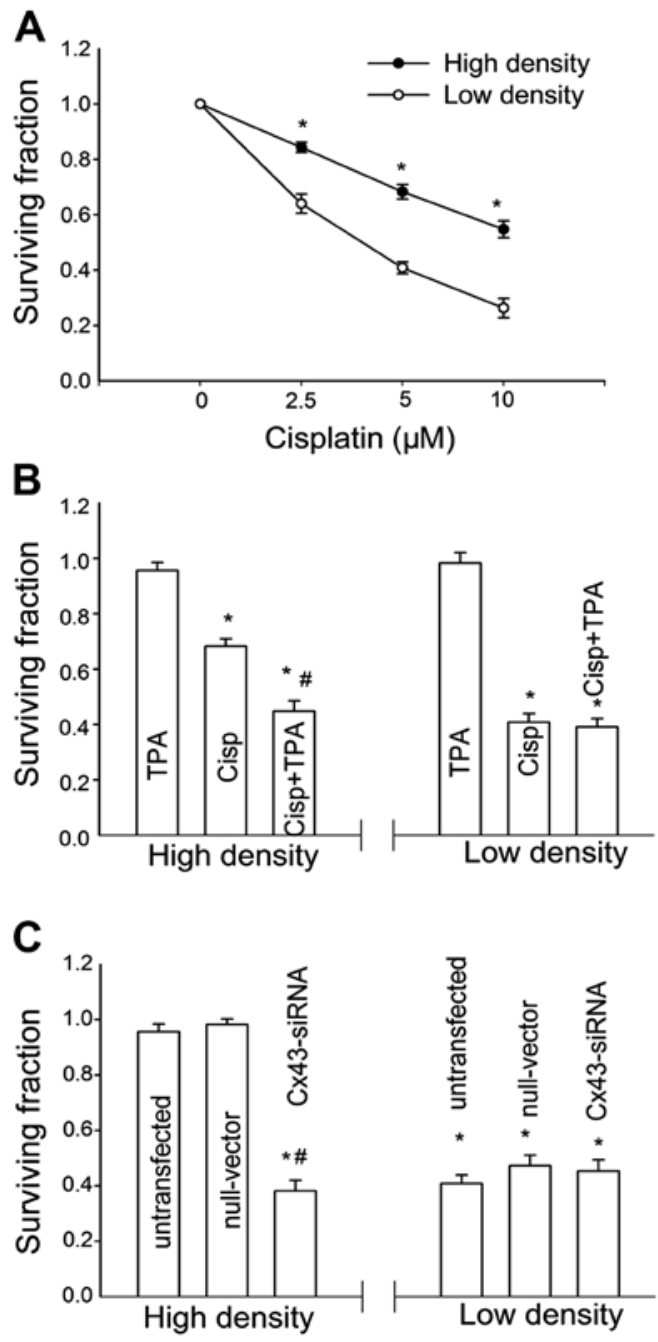

Figure 1. The effect of cell density on cisplatin cytotoxicity in TM4 cells is mediated by gap junction. (A) Clonogenic survival of cells treated with a range concentrations of cisplatin at a low cell density $(-\circ-, 100$ cells $/ \mathrm{ml})$ and high cell density $\left(-\bullet-, 3 \times 10^{4}\right.$ cells/ml). Points, mean for five to seven experiments; bars, SEM. *, Significantly different from low cell density group, $\mathrm{P}<0.05$. (B) Clonogenic survival of cells treated with $5 \mu \mathrm{M}$ cisplatin with or without $50 \mathrm{nM}$ TPA at low and high cell density. (C) Clonogenic survival of siRNA-transfected and untransfected cells treated with $5 \mu \mathrm{M}$ cisplatin at low and high cell density. Columns, mean for five experiments; bars, SEM. *, Significantly different from control, $\mathrm{P}<0.05$; \#, significantly different from the cisplatin bar in (B) and from the untransfected cells bar in (C), $\mathrm{P}<0.05$.

reduction in survival fraction from 68 to $43 \%$. By contrast, TPA had no effect on cisplatin cytotoxicity when the cells were cultured at a low cell density (Fig. 1B). Cx43 protein expression and junctional coupling were downregulated in TM4 cells stably transfected with Cx43 siRNA (Fig. 2). Consistent with the TPA results, cisplatin toxicity in siRNA-transfected cells was greater than that of the untransfected cells at high cell density. At a low cell density, cisplatin responses showed no differences between siRNA-transfected and -untransfected cells (Fig. 1C). These results suggested that inhibition of GJIC substantially increased cisplatin cytotoxicity only at high density, suggesting the density-dependence of cisplatin cytotoxity in TM4 cells is mediated by gap junctions.

Simvastatin reduces cisplatin cytotoxicity in TM4 cells. We examined the effect of simvastatin on cisplatin cytotoxicity in 

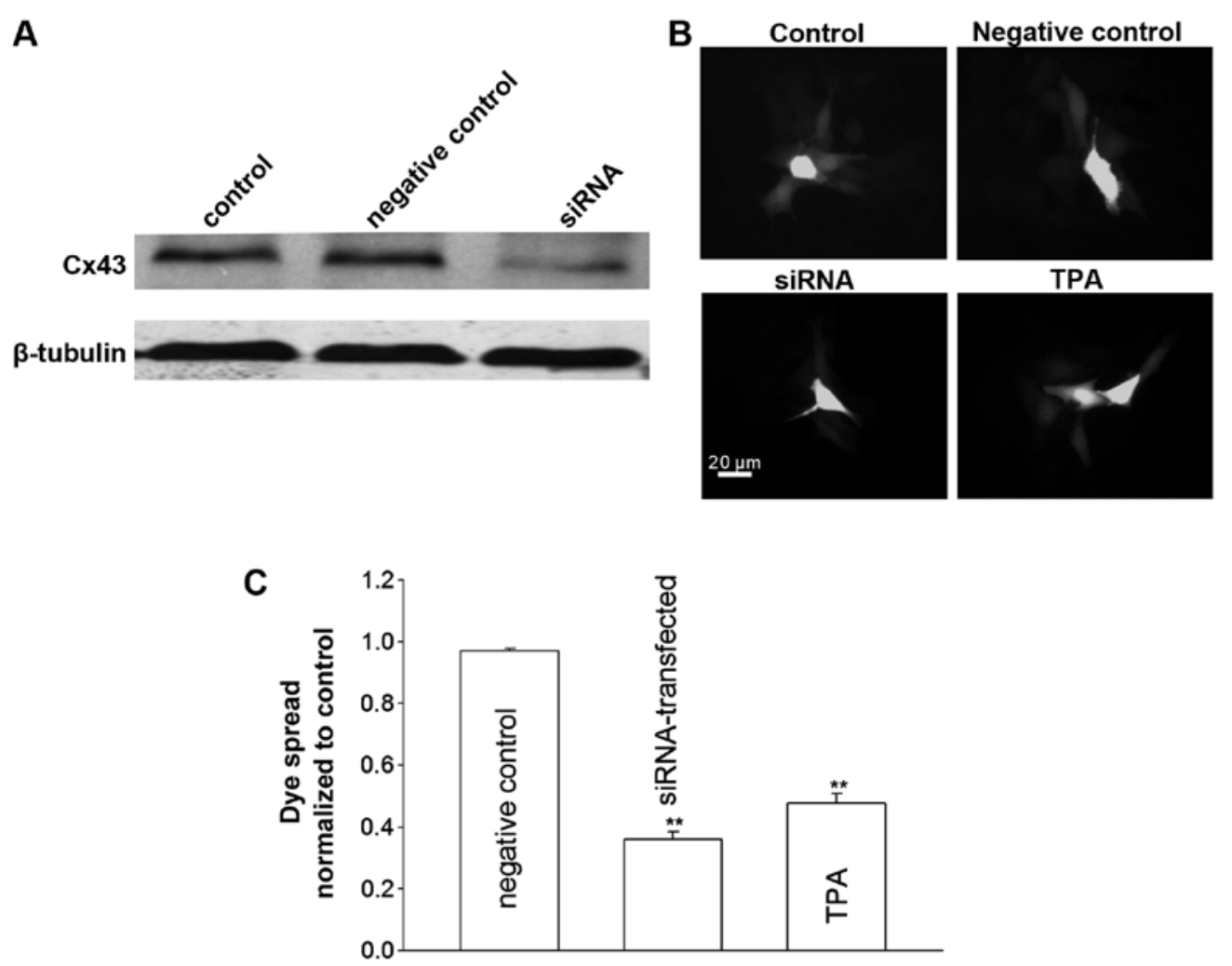

Figure 2. Inhibition of junctional communication by $\mathrm{Cx} 43$ siRNA and pharmacological inhibitor TPA. (A) Western blotting probed with anti-Cx43 antibody shows the decrease of the $\mathrm{Cx} 43$ level in siRNA-transfected cells. (B and C) Cx43-siRNA and TPA reduce dye coupling through gap junction as measured by parachute dye-coupling assay. Columns, mean for five experiments; bars, SEM. **, Significantly different from control, $\mathrm{P}<0.01$.

TM4 cells. TM4 cells were pretreated with $10 \mu \mathrm{M}$ simvastatin for $3 \mathrm{~h}$ and exposed to simvastatin and cisplatin for another 1 h. As shown in Fig. 3A, simvastatin itself had no toxic effect on TM4 cells at high and low density. At high density, the inhibition of clonogenic survival by cisplatin was attenuated by simvastatin. However, simvastatin exerted no effect on cisplatin toxicity at low density. As shown in Fig. 3B the simvastatin-induced suppression of cisplatin toxicity in TM4 cells was concentration-dependent. The cytotoxicity of cisplatin was reduced with increasing concentrations of simvastatin.

Simvastatin-induced suppression of cisplatin cytotoxicity is reversed by GJIC reduction. To investigate whether the density-dependent simvastatin effects were mediated by GJIC, Cx43-siRNA stably transfected cells were used. In Fig. 3C, simvastatin-induced reduction in cisplatin toxicity was markedly attenuated in Cx43-siRNA stably transfected cells at a high density. At low cell density where gap junction rarely formed, there was no difference between the control and Cx43-siRNA-transfected cells. These results indicated that the simvastatin-induced decrease of cisplatin cytotoxicity was reversed by GJIC reduction.

Effect of simvastatin on GJIC and Cx43 membrane localization in TM4 cells. The results described above support that the inhibitory effect of simvastatin on cisplatin cytotoxicity requires functional gap junctions, indicating that simvastatin may regulate gap junction activity. At high cell density when gap junction is formed, simvastatin may enhance GJIC, which mediated the transfer of possible protection signals among normal tesicular cells thereby reducing cisplatin toxicity. To confirm this hypothesis, we examined the effect of simvastatin on junctional coupling between TM4 cells. Fig. 4A shows that the cells that have been treated with simvastatin for $4 \mathrm{~h}$ have a marked increase in calcein dye diffusion through gap junctions. The dye coupling was gradually enhanced with increasing concentrations of simvastatin from 0.5 to $25 \mu \mathrm{M}$.

Immunofluorescent staining was used to examine whether simvastatin-enhanced GJIC occurred due to an increase in functional gap junction proteins on plasma membrane. In Fig. 4B, Cx43 was observed at appositional plasma membranes between coupled TM4 cells. Treatment with simvastatin to TM4 cells for 30 min or up to $4 \mathrm{~h}$ induced a great increase in Cx43 immunostaining density in the plasma membrane regions. These results suggested that simvastatin enhanced GJIC in TM4 cells by increasing the distribution of constituent $\mathrm{Cx} 43$ on the plasma membrane.

Simvastatin inhibits PKC-mediated CX43 phosphorylation. In order to investigate the mechanism by which simvastatin enhanced GJIC, western blotting was performed to examine the expression of constituent $\mathrm{Cx} 43$ in simvastatin-treated cells. As shown in Fig. 5A, simvastatin did not alter the level of total Cx43.

Cx43 phosphorylation is known to play an important role in gap junction disassembly and internalization from the cell surface (17). Our previous study on Leydig tumor cells raises 



Figure 3. Simvastatin attenuates cisplatin toxicity at high cell density and this effect was blocked by Cx43-siRNA. (A) Clonogenic survival of cells pretreated with $10 \mu \mathrm{M}$ simvastatin for $3 \mathrm{~h}$, and co-incubated with $5 \mu \mathrm{M}$ cisplatin for $1 \mathrm{~h}$ at high and low cell density. (B) Clonogenic survival of cells pretreated with a range of concentrations of simvastatin for the indicated periods, followed by co-incubation with $5 \mu \mathrm{M}$ cisplatin for $1 \mathrm{~h}$ at high cell density. (C) Clonogenic survival of siRNA-transfected cells pretreated with $10 \mu \mathrm{M}$ simvastatin for $3 \mathrm{~h}$, and co-incubated with $5 \mu \mathrm{M}$ cisplatin for $1 \mathrm{~h}$ at high and low cell density. Columns, mean for five experiments; bars, SEM. ", Significantly different from control, $\mathrm{P}<0.05$; \#, significantly different from the cisplatin bar, $\mathrm{P}<0.05$.
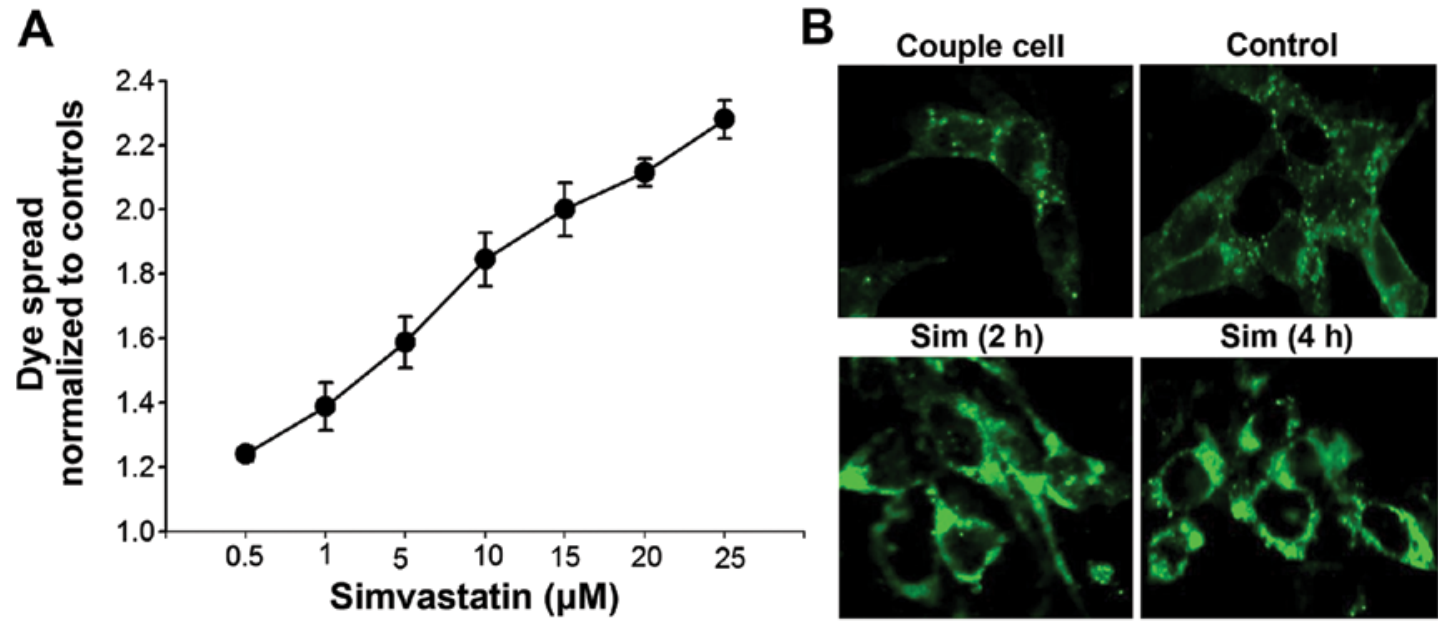

Figure 4. Effects of simvastatin on gap junction intercellular communication, Cx43 immunostaining. (A) The dye spread of cells exposed to increasing concentrations of simvastatin for $4 \mathrm{~h}$ as assayed by parachute dye-coupling assay. (B) Immunostaining for Cx43 in cells with or without $10 \mu \mathrm{M}$ simvastatin treatment. Magnification, $\mathrm{x} 400$.

the possibility of targeting PKC as a regulatory signal for simvastin to induce Cx43 dephosphorylation (15). We then investigated the effect of simvastatin on the phosphorylation status of Cx43. In Fig. 5B, the amount of phosphorylated Cx43 at serine 368 (ser368) which was identified to be a specific phosphorylation site for PKC was markedly reduced in cells pretreated with simvastatin. This reduction was enhanced with increasing simvastatin concentrations. The expression of phosphorylated Cx43 in Sertoli cells was almost 50\% of control after simvastatin treatment for $4 \mathrm{~h}$.

As shown in Fig. 6, simvastatin markedly reduced PKC expression. The decrease of PKC expression occurred at $30 \mathrm{~min}$ and peaked in $4 \mathrm{~h}$. Consistent with the alteration of PKC expression, PKC activity was also reduced in a timedependent manner in simvastatin-treated cells (Fig. 6B). To investigate that $\mathrm{PKC}$-mediated connexin phosphorylation 
A

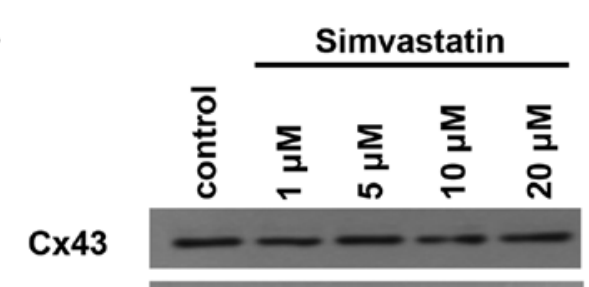

$\beta$-tubulin

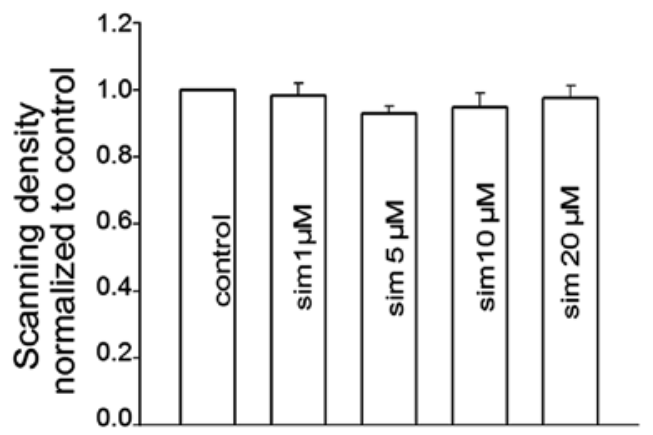

B
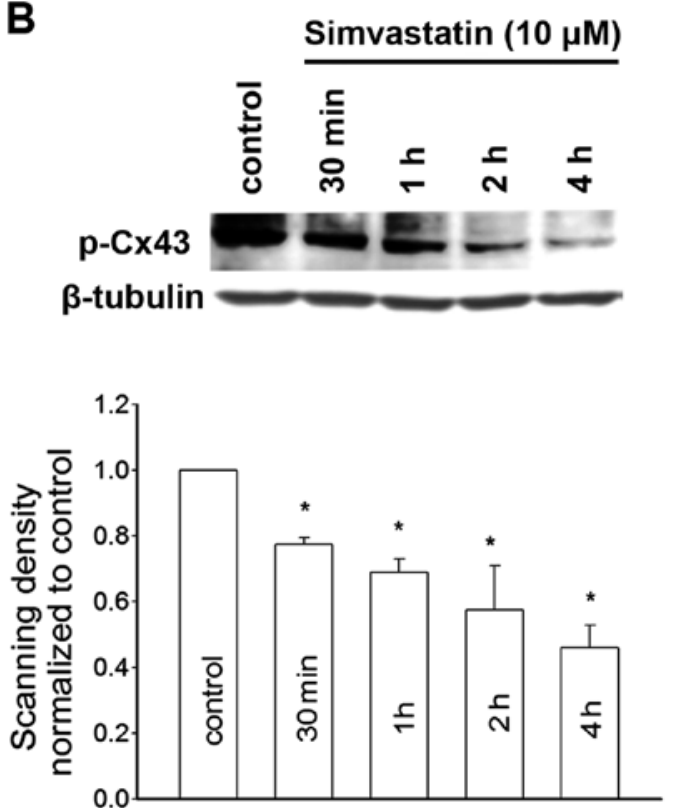

Figure 5. Effects of simvastatin on Cx43 expression and p-Cx43 (ser368) expression. (A) Western blot analysis of Cx43 expression in cells treated with varying concentrations of simvastatin. (B) Western blotting showing p-Cx43 (ser368) levels in cells following treatment with $10 \mu \mathrm{M}$ simvastatin for varying time periods. Bar graphs are derived from the densitometric scanning of the blots and shows a comparison of the level of Cx to $\beta$-tubulin density ratio. Columns, mean from four experiments; bars, SEM. *, Significantly different from control.

A
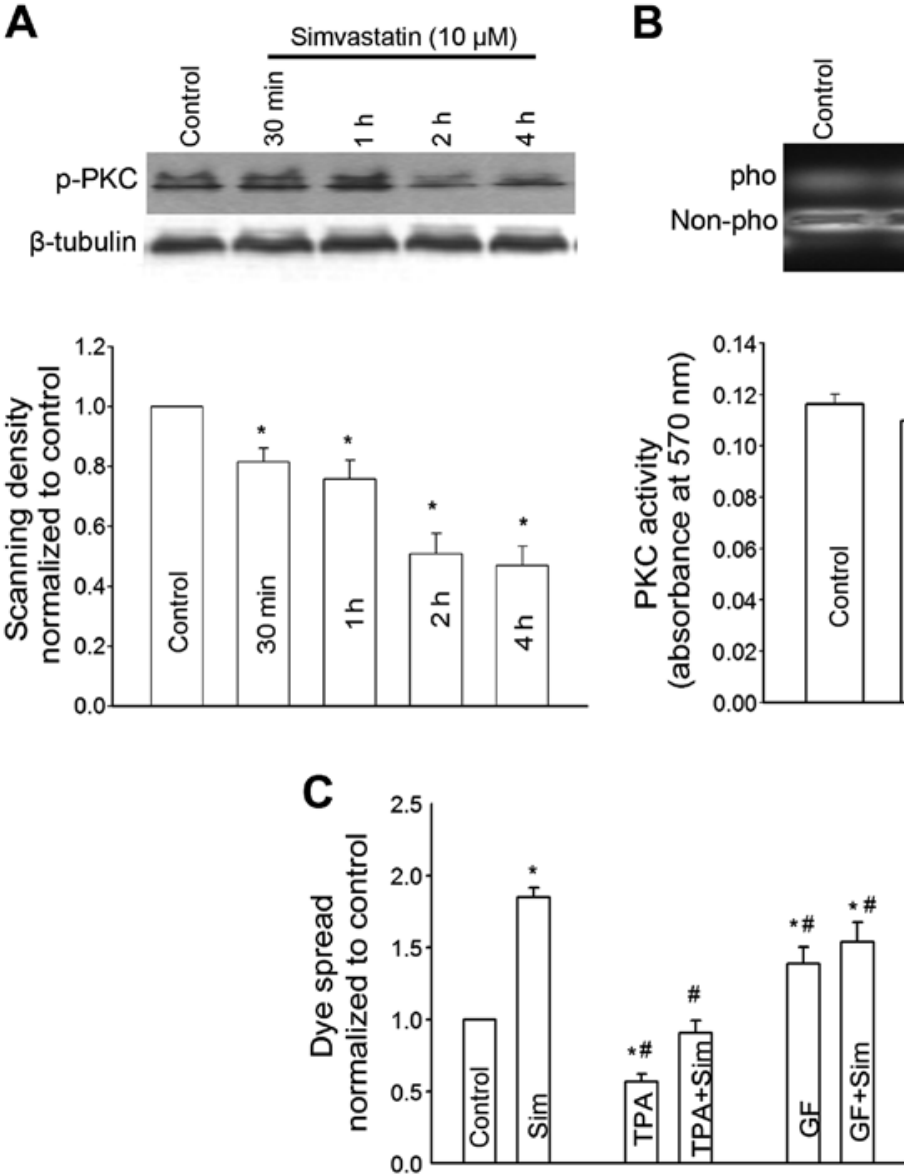

B
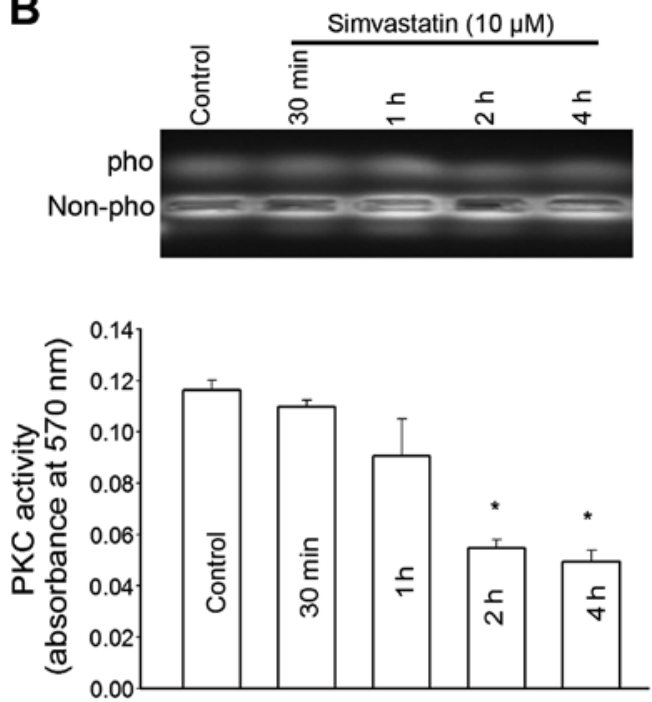

Figure 6. Role of PKC activity on simvastatin-induced enhancement of gap junction communication. (A) Western blotting showing levels of PKC in TM4 cells treated with $10 \mu \mathrm{M}$ simvastatin for the indicated time period. (B) The cells treated with simvastatin for the indicated time periods were subjected to a non-radioactive PKC assay described in Materials and methods. PKC activity is expressed as absorbance at a wavelength of 570 nm. (C) Comparison of dye spread through cells treated with GF109203X $(1 \mu \mathrm{M})$ or TPA $(50 \mathrm{nM})$ for $4 \mathrm{~h}$ with and without simvastatin (10 $\mu \mathrm{M})$. (A and B) Columns, mean from four experiments; bars, SEM. *, Significantly different from control. (C) Columns, mean for five experiments; bars, SEM. *, Significantly different from control, $\mathrm{P}<0.05$; ", significantly different from simvastatin bar, $\mathrm{P}<0.05$. 
was associated with the simvastatin-induced enhancement of GJIC, dye spread of Sertoli cells was then assessed in the cells treated with simvastatin and GF109203X, a specific PKC inhibitor or TPA, a PKC activator. GF109203X increased the dye spread of Sertoli cells through GJ, while TPA markedly decreased the dye spread. The simvastatin-induced increase of GJIC in Sertoli cells was slightly affected by simultaneous pretreatment with GF109203X. TPA exerted a significant suppression effect on the simvastatin-induced enhancement of dye spread through GJ.

\section{Discussion}

Infertility is one of the most serious late adverse consequences after cisplatin-based chemotherapy treatment $(2,18)$. Cisplatin induces toxicity in germ cells and in Sertoli and Leydig cells, and eventually results in long-lasting azoospermia and testicular atrophy in male adults (19). Disruption of the cell junction has been identified between Sertoli cells in rats after intraperitonal injection with cisplatin (20). Investigation into cultured cells has also demonstrated that cisplatin reduced the production of transferrin, androgen-binding protein, lactate and estradiol in Sertoli cells (21). In concordance with this observation, we found that cisplatin induced cytotoxicity in Sertoli cells, with clonogenic survivals decreasing $32 \%$ in controls after incubation with cisplatin for $1 \mathrm{~h}$.

The concentration of cisplatin used in the present study was lower relative to some in vitro models, but was approximately the peak plasma concentration during chemotherapy (22). Our paradigm allowed the GJIC-mechanism observed in the present study to be applied. Thus, a much higher concentration than that used in our text led to extensive cell death and no GJIC-mediated effects were identified. Investigators have shown that the inter-Sertoli cell junctions constituting the blood-testis barrier become leaky even at low-cisplatin doses (3). Clinically, normal cells are less sensitive to antitumor agents compared with cancer cells. Thus, long-lasting continuous cisplatin exposure in testicular tumor patients produces toxicity on Sertoli cells.

$\mathrm{Cx} 43$ is the predominately expressed $\mathrm{Cx}$ in testis. It is localized between Sertoli and Germ cells in seminiferous epithelium as well as in Leydig cells (23). Cx43 is not associated with the physiological functions of Leydig cells. However, Cx43 is critical for the formation of junctions and coordination of junctional communication between Sertoli and Germ cells and plays a vital role in the processes of Sertoli proliferation as well as Germ cell proliferation and differentiation (23). Cx43 in Sertoli cells is considered important in spermatogenesis (7). It has been revealed that $\mathrm{Cx} 43$ expression was significantly reduced in testes of infertile patients with secretory azoospermia. In Sertoli cell-only syndrome rat, Cx43 expression in Sertoli cells was undetected and the GJIC between them was impaired. Sridharan et al and Günther et al have confirmed that lack of $\mathrm{Cx} 43$ solely in Sertoli cells was sufficient to induce the arrest of spermatogenesis $(24,25)$. By contrast, enviroment chemicals have been found to affect Sertoli cell interactions through junctional proteins, and specifically $\mathrm{Cx} 43$ exerted deleterious effects on spermatogenesis (26). In light of those findings, an extensive exploitation of drugs or biologic approach to restore $\mathrm{Cx} 43$ expression and increase GJIC in Sertoli cells is of considerable value for improving reproduction. Our studies therefore suggest that future investigations in the putative therapeutic roles of simvastatin in decreasing reproduction toxicity among male patients should be performed.

Statins, classical cholesterol-lowering drugs, exert pleiotropic effects on osteoporosis, inflammation and other diseases generated by different issues $(27,28)$. Cx43 also plays essential roles in coordinating activities in the majority of organs. To the best of our knowledge, GJIC, which we demonstrated to be enhanced by simvastatin in the present study, seems to provide a probable mechanism as evidence for pleiotropic actions. An increase of GJ conductance protects heart from life-threatening arrhythmia (29). The antiarrhythmic ability of simvastatin thus seems due to its action on GJ (30). Furthermore, whether simvastatin-induced effects on GJIC ameliorate cisplatin-induced cardiovascular toxicity, which was also shown to be associated with $\mathrm{Cx} 43$ merit future investigation.

Phosphorylation is critically involved in Cx disassembly, degradation and internalization. It therefore altered the amounts of constituent Cxs localized on membrane and GJ function. $\mathrm{Cx} 43$ is the most easily phosphorylated Cx subtype, containing 12 or more serine or tyrosine residues at carboxyl terminal that can be extensively phosphorylated by a multiplicity of phosphorylation protein kinases $(17,31)$. Simvastatin is a lipophilic statin that permeates through plasma membrane by passive diffusion. It has been observed that simvastatin inhibits the activation of different kinases such as PKC or MAPKs in various cell culture $(15,32)$. Li et al focused on Sertoli cells, showing that PKC played a crucial role in regulating tight junctions during spermatogenesis (33). Our findings confirm that simvastatin induced a significant decrease in PKC activity and the simvastatin-induced increase of junctional dye transfer was abolished by TPA (a PKC activator) and imitated by GF109203X (a PKC inhibitor). Therefore, our results suggest that $\mathrm{PKC}$ is a regulatory signal for simvastin to induce Cx43 dephosphorylation in Sertoli cells.

The GJIC-mediated protection effect in normal cells has also been confirmed by other authors showing that neuronal vulnerability to oxidative stress and ischemia was significantly increased by the inhibition of astrocytic gap junctions $(11,34)$, and that $\mathrm{Cx} 43$ exerted a protective effect against oxidative stress-induced cell death in human retinal pigment epithelial cells and in cultured primary osteocytes $(10,35)$. Cx43 itself has been suggested to contribute to the activation of a major cytoprotective signaling pathway in cardiomyocytes (36). The cells exposed to cisplatin usually produce apoptosis by the formation of crosslinks, including intra- and inter-DNA crosslinks. It has also been demonstrated that cisplatin toxicity in rat liver epithelial cells was enhanced by GJIC (8). However, in normal testicular cells, cisplatin-induced DNA crosslinks were increased when GJIC was blocked by 18-GA or siRNA. GJIC is believed to communicate predominantly protective signals in normal testicular cells through gap junction in response to cisplatin (12). Nevertheless, which and how protective signals were triggered after cisplatin exposure have not been identified and whether it directly or indirectly induces the decrease of DNA crosslinks remains to be investigated. Glutathione is speculated to be a likely candidate. 
Glutathione $(\mathrm{GSH})$ is a tripeptide with a molecular weight of $307 \mathrm{Da}$ and is permeable to gap junctions. As reported by Nakamura et al (37), metabolic coupling of GSH between mouse and quail myocytes through gap junctions played an essential role in resistance of mouse myocytes to oxidative stress. Hepatotoxicity induced by acetaminophen was more higher in $\mathrm{Cx} 32-\mathrm{KO}$ mice and this was associated with a lower level of cellular GSH concentration. That study identified the protective effect may be due to GSH transmission between neighboring cells through GJIC (38).

To the best of our knowledge, the present study is the first to demonstrate that the enhancement effect of simvastatin on GJ has a protective effect against cisplatin-induced toxicity on Sertoli cells. The beneficial role of GJ in cisplatin-based chemotherapy therefore is bidirectional. An increase of GJIC by simvastatin has synergistically toxic effects on tumor cells, while the increase of GJIC on Sertoli cells alleviated their sensitivity to antineoplastic agents and therefore improved reproductive potency in male adults. The present study provides an update on the pharmacologic intervention of GJ or connexins and highlights the importance of basic cell biology in decreasing reproduction toxicity caused by exposure to chemotherapy.

\section{Acknowledgements}

The present study was supported by a grant from the National Natural Science Foundation of China (nos. 81401017, 81373439 and U1303221).

\section{References}

1. Elzinga-Tinke JE, Dohle GR and Looijenga LH: Etiology and early pathogenesis of malignant testicular germ cell tumors: Towards possibilities for preinvasive diagnosis. Asian J Androl 17: 381-393, 2015.

2. Brydøy M1, Fosså SD, Klepp O, Bremnes RM, Wist EA, WentzelLarsen T, Dahl O; Norwegian Urology Cancer Group III study group: Paternity and testicular function among testicular cancer survivors treated with two to four cycles of cisplatin-based chemotherapy. Eur Urol 58: 134-140, 2010.

3. Efstathiou E and Logothetis CJ: Review of late complications of treatment and late relapse in testicular cancer. J Natl Compr Canc Netw 4: 1059-1070, 2006.

4. He B, Tong X, Wang L, Wang Q, Ye H, Liu B, Hong X, Tao L and Harris AL: Tramadol and flurbiprofen depress the cytotoxicity of cisplatin via their effects on gap junctions. Clin Cancer Res 15: 5803-5810, 2009.

5. Shishido SN and Nguyen TA: Gap junction enhancer increases efficacy of cisplatin to attenuate mammary tumor growth. PLoS One 7: e44963, 2012.

6. Saez JC, Berthoud VM, Branes MC, Martinez AD and Beyer EC: Plasma membrane channels formed by connexins: Their regulation and functions. Physiol Rev 83: 1359-1400, 2003.

7. Weider K, Bergmann M and Brehm R: Connexin 43: Its regulatory role in testicular junction dynamics and spermatogenesis. Histol Histopathol 26: 1343-1352, 2011.

8. Jensen R and Glazer PM: Cell-interdependent cisplatin killing by $\mathrm{Ku} / \mathrm{DNA}$-dependent protein kinase signaling transduced through gap junctions. Proc Natl Acad Sci USA 101: 6134-6139, 2004.

9. Contreras JE, Sánchez HA, Véliz LP, Bukauskas FF, Bennett MV and Sáez JC: Role of connexin-based gap junction channels and hemichannels in ischemia-induced cell death in nervous tissue. Brain Res Brain Res Rev 47: 290-303, 2004.

10. Kar R, Riquelme MA, Werner S and Jiang JX: Connexin 43 channels protect osteocytes against oxidative stress-induced cell death. J Bone Miner Res 28: 1611-1621, 2013.
11. Nakase T, Fushiki S and Naus CC: Astrocytic gap junctions composed of connexin 43 reduce apoptotic neuronal damage in cerebral ischemia. Stroke 34: 1987-1993, 2003.

12. Hong X, Wang Q, Yang Y, Zheng S, Tong X, Zhang S, Tao L and Harris A: Gap junctions propagate opposite effects in normal and tumor testicular cells in response to cisplatin. Cancer Lett 317: 165-171, 2012.

13. Subramanian S, Emami H, Vucic E, Singh P, Vijayakumar J, Fifer KM, Alon A, Shankar SS, Farkouh M, Rudd JH, et al: High-dose atorvastatin reduces periodontal inflammation: A novel pleiotropic effect of statins. J Am Coll Cardiol 62: 2382-2391, 2013.

14. Hwang KE, Kwon SJ, Kim YS, Park DS, Kim BR, Yoon KH, Jeong ET and Kim HR: Effect of simvastatin on the resistance to EGFR tyrosine kinase inhibitors in a non-small cell lung cancer with the T790M mutation of EGFR. Exp Cell Res 323: 288-296, 2014.

15. Wang L, Fu Y, Peng J, Wu D, Yu M, Xu C, Wang Q and Tao L: Simvastatin-induced up-regulation of gap junctions composed of connexin 43 sensitize Leydig tumor cells to etoposide: An involvement of PKC pathway. Toxicology 312: 149-157, 2013.

16. Koreen IV, Elsayed WA, Liu YJ and Harris AL: Tetracyclineregulated expression enables purification and functional analysis of recombinant connexin channels from mammalian cells. Biochem J 383: 111-119, 2004.

17. Laird DW: Connexin phosphorylation as a regulatory event linked to gap junction internalization and degradation. Biochim Biophys Acta 1711: 172-182, 2005.

18. Boekelheide K: Mechanisms of toxic damage to spermatogenesis. J Natl Cancer Inst Monogr 34: 6-8, 2005.

19. Harman JG and Richburg JH: Cisplatin-induced alterations in the functional spermatogonial stem cell pool and niche in C57/BL/6 J mice following a clinically relevant multi-cycle exposure. Toxicol Lett 227: 99-112, 2014.

20. Pogach LM, Lee Y, Gould S, Giglio W, Meyenhofer M and Huang HF: Characterization of cis-platinum-induced Sertoli cell dysfunction in rodents. Toxicol Appl Pharmacol 98: 350-361, 1989.

21. Nambu A, Kumamoto Y and Mikuma N: Effects of anti-cancer agents on cultured rat Sertoli cells. Nihon Hinyokika Gakkai Zasshi 86: 1132-1136, 1995 (In Japanese).

22. Erdlenbruch B, Nier M, Kern W, Hiddemann W, Pekrun A and Lakomek M: Pharmacokinetics of cisplatin and relation to nephrotoxicity in paediatric patients. Eur J Clin Pharmacol 57: 393-402, 2001.

23. Carette D, Weider K, Gilleron J, Giese S, Dompierre J, Bergmann M, Brehm R, Denizot JP, Segretain D and Pointis G: Major involvement of connexin 43 in seminiferous epithelial junction dynamics and male fertility. Dev Biol 346: 54-67, 2010.

24. Sridharan S, Simon L, Meling DD, Cyr DG, Gutstein DE, Fishman GI, Guillou F and Cooke PS: Proliferation of adult Sertoli cells following conditional knockout of the Gap junctional protein GJA1 (connexin 43) in mice. Biol Reprod 76: 804-812, 2007.

25. Günther S, Fietz D, Weider K, Bergmann M and Brehm R: Effects of a murine germ cell-specific knockout of connexin 43 on connexin expression in testis and fertility. Transgenic Res 22: 631-641, 2013.

26. Qiu L, Zhang X, Zhang X, Zhang Y, Gu J, Chen M, Zhang Z, Wang $X$ and Wang SL: Sertoli cell is a potential target for perfluorooctane sulfonate-induced reproductive dysfunction in male mice. Toxicol Sci 135: 229-240, 2013.

27. van der Meij E, Koning GG, Vriens PW, Peeters MF, Meijer CA, Kortekaas KE, Dalman RL, van Bockel JH, Hanemaaijer R, Kooistra T, et al: A clinical evaluation of statin pleiotropy: Statins selectively and dose-dependently reduce vascular inflammation. PLoS One 8: e53882, 2013.

28. Uzzan B, Cohen R, Nicolas P, Cucherat M and Perret GY: Effects of statins on bone mineral density: A meta-analysis of clinical studies. Bone 40: 1581-1587, 2007.

29. Greener ID, Sasano T, Wan X, Igarashi T, Strom M, Rosenbaum DS and Donahue JK: Connexin43 gene transfer reduces ventricular tachycardia susceptibility after myocardial infarction. J Am Coll Cardiol 60: 1103-1110, 2012.

30. Naji F, Suran D, Kanic V, Vokac D and Sabovic M: Comparison of atorvastatin and simvastatin in prevention of atrial fibrillation after successful cardioversion. Int Heart J 50: 153-160, 2009. 
31. Aravindakshan $\mathbf{J}$ and Cyr DG: Nonylphenol alters connexin 43 levels and connexin 43 phosphorylation via an inhibition of the p38-mitogen-activated protein kinase pathway. Biol Reprod 72: 1232-2140, 2005.

32. Lee YM, Chen WF, Chou DS, Jayakumar T, Hou SY, Lee JJ, Hsiao G and Sheu JR: Cyclic nucleotides and mitogen-activated protein kinases: Regulation of simvastatin in platelet activation. J Biomed Sci 17: 45, 2010.

33. Li JC, Mruk D and Cheng CY: The inter-Sertoli tight junction permeability barrier is regulated by the interplay of protein phosphatases and kinases: An in vitro study. J Androl 22: 847-856, 2001.

34. Le HT, Sin WC, Lozinsky S, Bechberger J, Vega JL, Guo XQ, Sáez JC and Naus CC: Gap junction intercellular communication mediated by connexin 43 in astrocytes is essential for their resistance to oxidative stress. J Biol Chem 289: 1345-1345, 2014.
35. Pocrnich CE, Shao Q, Liu H, Feng MM, Harasym S, Savage M, Khimdas S, Laird DW and Hutnik CM: The effect of connexin43 on the level of vascular endothelial growth factor in human retinal pigment epithelial cells. Graefes Arch Clin Exp Ophthalmol 250: $515-522,2012$

36. Rottlaender D, Boengler K, Wolny M, Michels G, EndresBecker J, Motloch LJ, Schwaiger A, Buechert A, Schulz R, Heusch G, et al: Connexin 43 acts as a cytoprotective mediator of signal transduction by stimulating mitochondrial $\mathrm{K}_{\text {ATP }}$ channels in mouse cardiomyocytes. J Clin Invest 120: 1441-1453, 2010

37. Nakamura TY, Yamamoto I, Kanno Y, Shiba Y and Goshima K: Metabolic coupling of glutathione between mouse and quail cardiac myocytes and its protective role against oxidative stress. Circ Res 74: 806-816, 1994.

38. Igarashi I, Maejima T, Kai K, Arakawa S, Teranishi M and Sanbuissho A: Role of connexin 32 in acetaminophen toxicity in a knockout mice model. Exp Toxicol Pathol 66: 103-110, 2014. 